\title{
Using Heterotopias to Characterise Interactions in Physical/Digital Spaces
}

\author{
AKMAL Haider $\mathrm{Ali}^{\mathrm{a}^{*}}$ and COULTON Paul ${ }^{\mathrm{b}}$ \\ ${ }^{a}$ Imagination, Lancaster Institute for the Contemporary Arts, LICA Building, Lancaster University, UK, LA1 4YW. \\ ${ }^{b}$ Imagination, Lancaster Institute for the Contemporary Arts, LICA Building, Lancaster University, UK, LA1 4YW. \\ * Corresponding author e-mail: h.a.akmal@lancaster.ac.uk \\ doi: $10.21606 /$ dma.2017.348
}

This paper addresses the complexity of designing interactions in hybrid digital/physical spaces, in which notions of public and private are becoming increasingly blurred, by using a philosophical lens to characterise such spaces. In particular it references the ideas presented by Michel Foucault in his essay "Of Other Spaces". It proposes the presence of a spatial division within physical and virtual, in terms of private and public, and juxtaposes them through a Heterotopical Model for Inter-Spatial Interaction through which designers can examine the coexistence of physical and digital interactions. The purpose of modelling this juxtaposition is to help designers understand the nature of connections that happen between physical and digital objects in these spaces and consider how meaningful interactions can respond to this complexity.

spaces; phenomenology; heterotopia; philosophy for design

\section{Introduction}

This paper illustrates how philosophical constructs should not be viewed as separate from design practice but rather can augment the design process using the example of how to characterise complex interactions that combine both the physical and digital aspects. We establish the presence of a philosophical division of space developed through the ideas presented by Michel Foucault in his essay "Des Espace Autres" (Of Other Spaces). This division plays a pivotal role in the creation of a framework for Inter-Spatial Interaction, acting as a philosophical lens through which we define interactions between physical and digital aspects that traverse over an imagined Real Space and Digital Space. Referencing Foucault's idea of the heterotopia as the basis of this lens, we systematically define the presence of these alternate spaces and the nature of interactivity that could happen within them. Finally, a discussion is presented on the potential for designers to use this approach to understand the complex nature of objects that connect with digital interfaces and services in order to consider the "range of perspectives from which each device may be observed" 
(Lindley \& Coulton, 2017) and thus comprehend the "complexity relating to the interdependence" between interactions, raising questions on the need for 'meaningful interactions' between physical and digital in such environments.

\subsection{The division of Space}

Space is described by Tuan (1977) as, "an abstract term for a complex set of ideas", which he says comes from how, "people of different cultures differ in how they divide up their world, assign values to its parts, and measure them". His definition assumes space in relation to the "intimate experience [of man] with his body and with other people", wherein one, "organises space so that it conforms with and caters to his biological needs and social relations". Architecturally space is seen through an idea of dimensionality, where it can be measured, yet "spatial dimensions such as vertical and horizontal, mass and volume are experiences known intimately to the body" (Tuan, 1977, p. 108), this allows architecture to traverse the boundary between space and place. Both terms "denote common experiences" (Tuan, 1977, p. 3) but they both expand on each other's definitions where "place is security, space is freedom: we are attached to the one and long for the other". Tuan's exploration of space/place is more towards the study and experience of Geography, but it can be appropriated to encompass the digital as we have done within the research, as such:

Consider the sense of an 'inside' and an 'outside', of intimacy and exposure, of private life and public space. People everywhere recognise these distinctions, but the awareness may be quite vague (Tuan, 1977, p. 107)

The level of interaction a person might have within an open town square compared to their own house would be very different, as different amounts of trust would be associated with these 'inside' and 'outside' spaces. This space/place relationship transcends into our digital environments equally, with the "conceiving of cyberspace as a social space" (Slane, 2007, p. 12), it can be seen as being, "socially constructed, its meaning deriving from the uses to which it is put, and therefore capable of multiple simultaneous incarnations" - the word social here is taken liberally to include not only person to person interaction but also thing to thing interaction where digital terminals and objects would be included -therefore, a mobile phone would constitute as a personal space where as a message board online would be a public space only juxtaposed into a virtual world but in either case the interaction happens through a physical interface; here a mobile, or a laptop. These incarnations of digital spaces become more convoluted when imagining the plethora of Internet powered devices available, often with the Smart moniker preceding them; Smart Phones, Smart Watches, Smart TV's, and so on. A cluster of communications that have us "entangled within the heterogeneous network of interconnected objects or things that are readable, recognisable, locatable, addressable, and/or controllable via the Internet" (Coulton, 2015; Lindley, Coulton, \& Cooper, 2017). A space can thus have multiple places residing within it, each with its own meaning which is unique to the actors interacting within them.

Often these virtual interactions tend to mimic older real-world practices; a diary can exist in a physical and virtual form, both can be closed or open to others. The complexity ensues when multiple points of interaction come in to play with objects connected to wider constellations of interactions for instance when a Smart Assistant such as Google Home needs to connect with a mobile phone or a switch among multiple other points in order to request access and gaining trust to switch on a light bulb, these raise questions such as: what is the nature of these interactions? Are they meaningful for the actors? And how can one better design them to be not only efficient but also worthwhile?

\section{Methodology}

Phenomenological research attempts to understand, "how people experience things and events", by examining, "perspectives and views of various social realities" (Leedy \& Ormrod, 2010; Muratovski, 2015, p. 79). The Stanford Encyclopaedia of Philosophy defines phenomenology as the study of, 
"things as they appear in our experience, or the ways we experience things, thus the meanings things have in our experience" (Smith, 2016) and expresses an interest in a "conscious experience as experienced from the subjective or first-person point of view". Philosophical approaches such as speculative realism, or object-oriented ontology put aside old philosophical dualisms and instead explore how objects "should be recognised for their indifference to us" (Cole, 2013, p. 106) and focus on the things they do "behind our backs" looking at their individual experiences as "actants", moving in and out of "assemblages, entering into collectives of their own making". Therefore, by seeing these interactions existing as a phenomenon we attempt to make sense of their complexity using philosophical references in tandem with real-life examples. By asking, "What is it like to do or experience [something]?'” (Muratovski, 2015, p. 79), we attempt to empathise with these objects and see from their perspective what these Inter-Spatial Interactions are like.

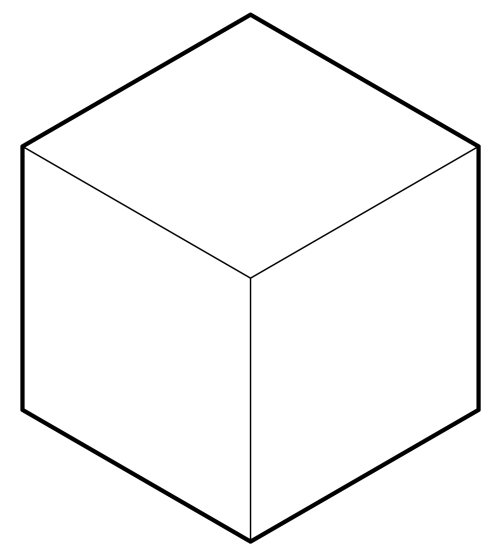

Real Space

Physical Interactions with physical interfaces such as a mobile phone or a switch

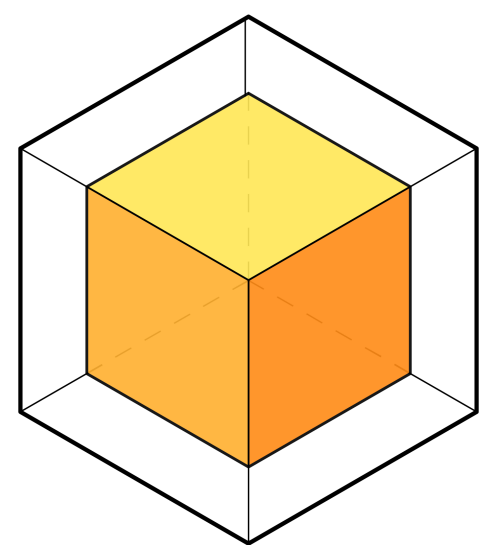

Digital Space

Digital Interactions with digital interfaces

such as a website server or applications

Figure 1. Imagining Digital Space as a subset of Real Space

For this philosophical lens, the actual space will be divided utilising Tuan's (1977) perspective of spaces containing a "sense of an 'inside', and an 'outside'", by presenting two realities; one being the physical reality that we have around us in which we physically interact (Real-Space or RS), the other being a virtual one where interactions through/with digital objects occur (Digital-Space or DS) (see Fig. 1). In this particular view DS resides as an 'inside' or a subset of RS, allowing for physical objects to be present within the same space alongside their virtual counterparts; one being tangible the other intangible. The idea of virtual being present alongside the physical has been discussed by others seeing it as a "Virtuality Continuum" (Milgram, Takemura, Utsumi, \& Kishino, 1995), one where, "both the real and the virtual coexist" (Coulton, 2017). Virtual worlds are also seen as literal places that, "can be construed not just in terms of globalised online networks, but in terms of space, landscape, and localities as well" (Rymarczuk \& Derksen, 2014). Descartes' explored the concept of a mind/body split which he called res extensa (extending things) and res cogitans (thinking things), commonly used to imagine the "physical world as having both extension and location in space" (Monk, 1997, p. 46), looking at psychological realities such as the virtual through this approach they, "do not have spatial dimensions, and their location is only metaphorically 'in the mind"'; therefore, the division of space can be justified through a philosophical embodiment of the virtual space as a similar yet altered parallel space to the physical residing within it.

The second step is to further characterise these spaces with their 'insides' and 'outsides', and in this particular case to consider: spheres of Private and Public. These spheres house information with which we physically and/or virtually interact with. As an example, take a public message board 
online to be analogous with a discussion in the park where others may very well hear you, similarly a personal passcode protected digital diary can be related with a physical key and lock diary. Although this is only in terms of the immediate relationship one has with objects and spaces around the objects and as we shall see for connected objects these interactions become considerably more complex.

\subsection{Grounding the Philosophy}

Having acknowledged the spaces, we now see specific overlaps happening between spaces and spheres (see Fig. 2). Foucault once said: "What is interesting is always interconnection, not the primacy of this over that" (Brooker, 1999), keeping that in mind we come to the philosophical basis of this paper. In his essay "Des Espace Autres" (Of Other Spaces) Foucault (1967) introduced the concept of the heterotopia exploring how our lives are "governed by a certain number of oppositions that remain inviolable", calling them "simple givens", being, "between family space and social space, between cultural space and useful space", but more importantly, "between private space and public space" (Foucault, 1967, p. 2). He explains these as ideals that are "nurtured by the hidden presence of the sacred" and calls these heterotopias placeless places because of their deviation from the norm. He goes on to assert that, "we do not live in a kind of void, inside of which we could place individual and thing" (p. 3), rather, "we live inside a set of relations that delineates sites which are irreducible to one another and absolutely not superimposable on one another". These other spaces thus exist as a, "simultaneously mythic and real contestation of the space in which we live". For Foucault, "Heterotopias are places of Otherness, whose Otherness is established through a relationship of difference with other sites, such that their presence either provides an unsettling of spatial and social relations or an alternative representation of spatial and social relations" (Hetherington, 2002, p. 8). Hetherington (2002) explains on how these spaces are created saying that they, "bring together heterogeneous collections of unusual things" (p. 43)-the deviation from the norm - where they have no, "order established through resemblance". Furthermore, he discusses that what matters is the relationship seen "from the standpoint of another perspective, that allows a space to be seen as heterotopic".

This approach makes it safe to imagine unique interactions that exist within the overlaps of the Inter-Spatial Interactivity model as residing within a heterotopia-or a series of heterotopias. A grounding factor of these spaces is that in these, "places of Otherness", "unsettling juxtapositions of incommensurate "objects'" are established each contesting, "the way our thinking is ordered" (Hetherington, 2002, p. 42); hence presenting an alternate ordering of things that is unsettling because they have "the effect of making things appear out of place" (Hetherington, 2002, p. 50). This particular aspect allows us to view interactions in these spaces in a manner of urgency and thus challenging their meaningfulness towards the actors and the act.

Although the concept of heterotopia has most commonly been used to define alternate physical spaces as those referenced by Foucault himself-such as the cemetery, a festival, or the library-it also is used to define more abstract structures as he explains with the, "rug [being] a sort of garden that can move across space" (Foucault, 1967, p. 6). Another analogy he gives is of the boat which he calls a, "heterotopia par excellence". Rymarczuk and Derksen (2014) discuss how the boat, "as a 'placeless place' applies to cyberspace as well, 'particularly when it is a network, linking terminals in different places and times into a unified environment'". They go on to assert through Sherman Young's point of view of how cyberspace can have [further] heterotopias as well".

\subsection{Principles of Heterotopia}

Foucault (1967) established six principles to explain his ideology of a heterotopia, to begin he affirms that all cultures display the ability to create, or have created, heterotopias though which they, "take quite varied forms" depending on causal relationships to the space they inhabit, the culture they are tethered to and other factors. Second, society has the ability to "guide, push, and make established heterotopias", in effect having of them, "change or adopt novel functions or new meanings" 
(Rymarczuk \& Derksen, 2014). Foucault explains this in relation to the cemetery which having evolved over time, "no longer the sacred and immortal heart of the city, but the other city, where each family possesses its dark resting place" (Foucault, 1967, p. 6). Third, is the "juxtaposing in a single real place are several spaces, several sites that are in themselves incompatible" (Foucault, 1967, p. 6). Rymarczuk and Derksen (2014) have expressed this to be a, "defining character of heterotopias", allowing the, "merg[ing] of certain spaces", such as public and private to exist. The fourth principle establishes a concept of heterochronies being that "heterotopias are most often linked to slices in time" (Foucault, 1967, p. 6), forcing an, "absolute break with traditional time"; cemeteries, museums, libraries, are examples of "becom[ing] heterotopias in which time never stops building up and topping its own summit". Fifth, "Heterotopias always presuppose a system of opening and closing that both isolates them and makes them penetrable" (Foucault, 1967, p. 7). This can be imagined through metaphorical gatekeepers entrusted with responsibilities to allow certain things to enter and exit the heterotopia, digitally this can be imagined through payment, registration, and identification protocols. Finally, heterotopias have, "a function in relation to all the space that remains" around them. Foucault (1967) defines this as a function that, "unfolds between two extreme poles", in a bid to, "expose every real space", through creating an alternate, "space of illusion", wherein defining a, "space of perfection to compensate for the flaws of real life" (Rymarczuk \& Derksen, 2014).

As an example of a digital space being a heterotopia, Rymarczuk and Derksen (2014) uses the example of Facebook, affirming how it requires actors or in its case, "user[s] follow rules of conduct", if they wish to, "start immersing themselves", in its virtual world and have to agree upon, "terms of agreement - a contract essentially stripping away all property claims of information posted within this space". They critique this aspect of the service saying that it is, "difficult to leave the space entirely"; recent updates of Facebook have added a deletion option though the design of the feature arguably discourages such activity which essentially aligns to the fifth principle of heterotopia. Moving on, they affirm that Facebook shows the, "distinct regime of time", that Foucault describes in his fourth principle comparing it to museums that "accumulate time", having it "share traits with but also combine them and add a dimension that marks it as an altogether new kind of heterochrony", summing up that, "Facebook collapses past life, present life and afterlife into something very other". They converge on the third principle by explaining how Facebook views privacy wherein the public domain, "is not invisible to the Facebook owners and administrators", and at the same time individual, "social spheres form one big network, owned and administrated by Facebook", and though individuals are divided into spaces, "the distinction between private and public does not hold", because, "Facebook as a whole is not an undivided space". Finally, for the sixth principle a discourse on the illusion that Facebook gives of connectivity which they, "characterise as a performance", and give power to, "inauthenticity", as people, "rejoice in the fact that it gives them the ability to present themselves to the world".

\subsection{A Model for Inter-Spatial Interactions}

These principles can just as well be established for physical devices that interact with-through RS and DS and while online services such as Facebook can on their own be seen as heterotopias, the following model is proposed to explore how physical and digital interactions can coexist in the same instance as a heterotopia. Figure 2 shows the proposed heterotopical model, inspired by Foucault's (1967) example of a mirror:

The mirror functions as a heterotopia in this respect: it makes this place that l occupy at the moment when I look at myself in the glass at once absolutely real, connected with all the space that surrounds it, and absolutely unreal, since in order to be perceived it has to pass through this virtual point which is over there (Foucault, 1967, p. 4)

He describes it as a parallel space which appears to have traits of a "utopia" since you see yourself where you are not; or as he places it "in an unreal, virtual space that opens up behind the surface" 
(p. 4)-here the seeing of oneself is taken in the sense of the actor in that space, so a mobile phone, or a toaster that can connect to the Internet can be imagined similarly. The act of seeing your activities on a Smart Phone, for example using Whatsapp, can also be understood from Foucaults example of the sounds on a telephone line which uses the same concept of the mirror analogy, wherein by talking to each other without being physically present in the same space and the hearing of each other's voice affirms their existence.

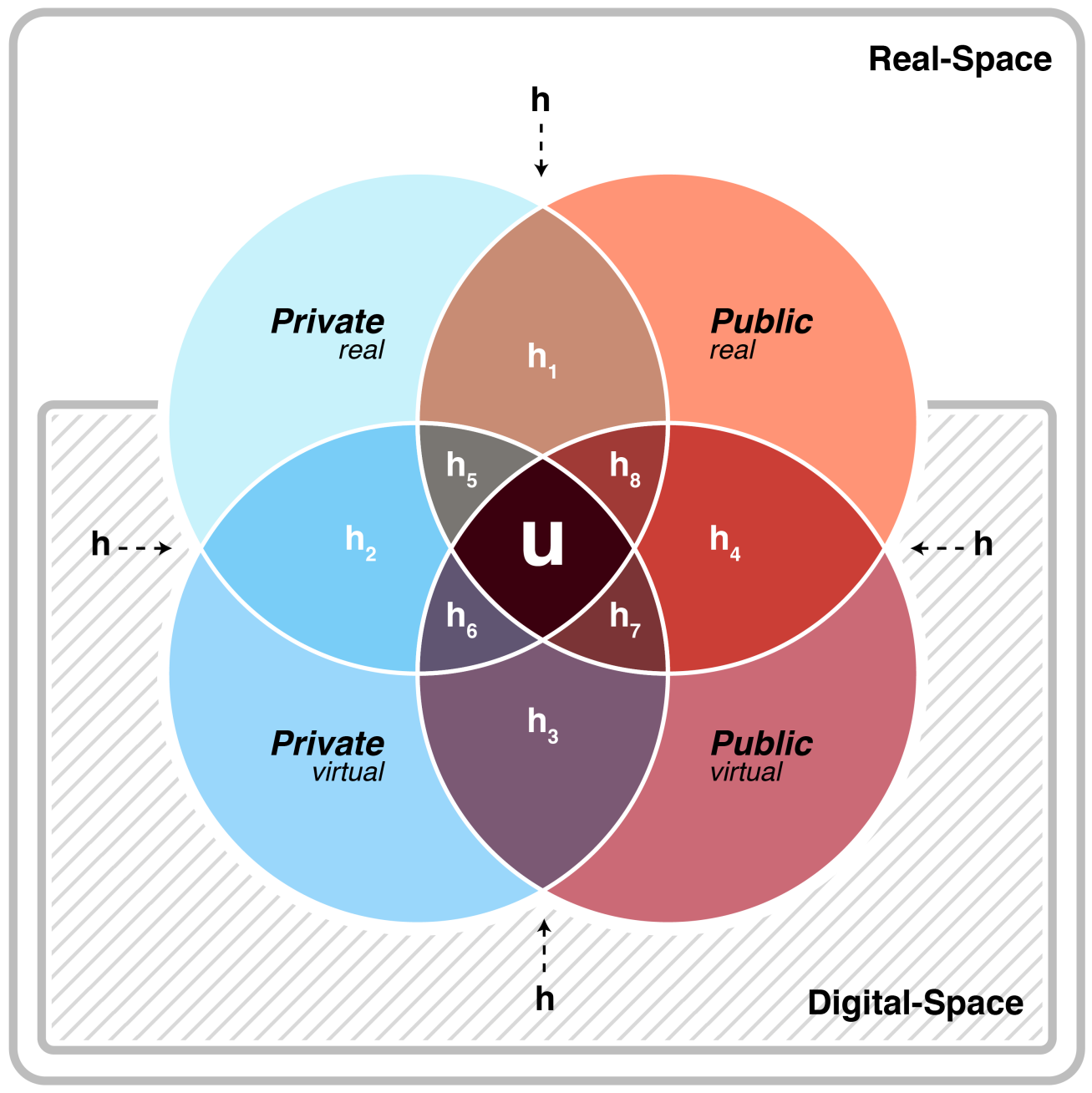

Figure 2. Philosophical Model for Inter-Spatial Interactivity

The model thus incorporates two spaces coexisting as one within the other each with its rules and regulations and encompassing individual spheres of privacy and publicity. The overlaps created can be characterised as: Private-Real (PrR), Public-Real (PuR), Private-Virtual (PrV), and Public-Virtual (PuV). Furthermore, overlaps are seen between the real and virtual iterations of privacy and publicity and they form the more unique and albeit complex heterotopias (h1 through h8).

Private-Real: One of the two divisions of RS, it encompasses ideals and information that are most intimate to us forming our inherent acknowledgement of the private. For instance, the physical space of a bedroom could be considered as a very real private space. Being a personal perspective it is hence of more importance to the individual to acknowledge it as such, but in order to function as a true 'private' it requires an understanding of a corresponding opposite;

Public-Real: Opposing general notions of privacy, it defines the private as much as it defines itself. An open reality that exists around us, governed by culture, society, government, policy, to name a few. The public exists as a platform of interaction that is open and valid for all to interfere/intersect 
with. Carrying on the example of a home, a communal living room could be accepted as real public space, and in a larger perspective a park where one can be easily seen and interacted with.

Private-Virtual: First of the two counterparts in DS, it incorporates rules that are defined by the individual to replicate their real notions of privacy. "The always-on, always-accessible network produces a broad set of changes to our concept of place" (Varnelis \& Friedberg, 2008). Referring to the mobile phone as a "telecocoon" Varnelis (2008) discusses how it "maintains intimacy at a distance, facilitating private encounters in public spaces", therefore creating the counter existence of the private in DS. A personal Smart Phone can be considered as a private virtual space within a physical object.

Public-Virtual: Second of the two counterparts this facilitates the public sphere through digital interfaces, Varnelis (2008) takes a cue from Jane Jacobs saying what "makes the public sphere vibrant is the continual contact with unexpected forms of interactions", the DS allows for a continuum of those interactions between Public-Real through to the virtual. A television can be seen as a virtual public space, one where interaction can be achieved through experiencing it, and since multiple people can experience the same thing together it allows for something akin to being at a concert.

Heterotopia 1: The first overlap to occur is between Real Private and Public spheres, here the interactions are those that happen in our daily physical lives influenced by very physical elements in the world around us. For the purpose of this paper and to aid understanding we will be using an example of fitness tracking to illustrate the differences within the model. An actor could imagine the physical steps they take as being a very physical private interaction that in truth is very public as the steps could be seen being taken by others in the same physical space. In both instances, the actor is in-charge of the act to happen becoming the gate-keeper, they take a step and in doing so have others potentially see it happen; an amount of time is accumulated in order to take each step and view it hence the acts are hetrochronies; each step being taken has an illusion of displacement which in this instance conform to the laws of physics and subsequently remove one from their initial stance (standing or moving) towards another.

Heterotopia 2: Moving clock-wise around the model shown in figure 2 the next overlap is seen between RS and DS, here using the same example of fitness tracking, this form can be seen when an actor uses a physical tracking device such as a FitBit to represent real steps in an alternate state, in this case numeric data. Although the information is the same, they both represent physical steps but due to the fact they are within two different spaces (RS and DS) they are visible in different ways. Variations of the Private clash together creating an alternate reality of privacy which exists only in DS hence it is in many ways similar to the illusion in Foucault's mirror; one version looks at the virtual version of themselves and grounds the others visibility in their own respective realms.

Heterotopia 3: Next we see an overlap between PrV and PuV, the interaction here should abide primarily by rules in the DS with little influence from RS. Continuing with our example, the steps saved to the fitness tracker are now allowed by the wearer to be saved to a server online. The reason this is a PuV interaction is because the server will be operated by other entities who could prescribe policies and regulations to oversee this information.

Heterotopia 4: The next overlap is between both iterations of public. Many interactions tend to exist in this space which are free to access through open data in order to create a publicly viable connection between the real and the virtual. Looking back at the steps taken example, imagine a wearable device that doesn't share data with its wearer but instead saves it immediately to a public server. A service such as If This Then That (IFTTT) could then be used to parse this data and initiate some action, for example, the step data is sent from the device directly then parsed into an online spreadsheet. Another way of considering this is through the example of a wifi light-bulb that's connected to a digital interface allowing you to turn it on or off via a mobile device. The bulb is in a room that can be operated through a public link on Facebook, anyone can access it and change the 
status of the physical bulb. The bulb exists as a physical object and has a digital presence accessible through the mobile device making it exist there as an alternate of itself. When turning the bulb on from the mobile there is no physical interaction being made with the bulb yet a very physical alteration occurs in the state of the bulb wherein it turns on. This makes this interaction a very public one where even though physical contact is not happening a very visible physical change occurs.

Heterotopia 5: The inner overlaps of the model are where more complicated interactions begin to appear governed according to orders. The first of which occurs as a PrR-PrV-PuR interaction. As this occurs primarily in $\operatorname{Pr} R$ it would be more influential but the interaction would have traits of the other spheres. Take our steps being saved from our FitBit, what if that data were to be synced with another device of another wearer and they could scroll through data that's been shared with them and vice versa? Although the information here is present in different versions (real steps and numeric iterations) the presence of another individual and their physical device can be taken as it being in both real and digital spaces

Heterotopia 6: Here we see a PrV-PrR-PuV overlap with things primarily grounded by the PrV but influenced by others. This can be imagined very similar to example in $\mathrm{H} 5$ but substituting the second device with a website where all data is synced and shared with a wider community. The use of social media can also be imagined here, your fitness tracker saves physical data it interacts with and sends that to a digital server which subsequently interacts with a social network such as Facebook and shares the information publically. The movement of this information from RS to DS and then again into DS but as a very different version of itself shows how simple data collection can be repurposed exponentially, with every jump changing the data to reaffirm according to the nature of the other space it inhabits.

Heterotopia 7: In a PuV-PrV-PuR overlap a more digital approach of trust can be observed. The IFTTT protocol earlier imagined to save data to a spreadsheet can be reconsidered, only this time instead of saving to a personal spreadsheet the data is visualised on a public device such as a digital display in an office telling all its employees about how many steps have been taken in the office only by the employees.

Heterotopia 8: Finally, in a PuR-PuV-PrR overlap one can see a physical dominating the virtual. A way to picture this interaction would be with a door that can monitor people going in and out of it using wearable RFID tags. The data is coming from a physical source and returning to a physical source by being displayed publicly but what makes this unique from the $\mathrm{H} 7$ is that here the data is taken directly from the physical source and not through any virtual channels, alternatively to make it more interesting, the PuV can be a source of information that could be syncing a particular individual according to their interaction with the door. So, imagine a shoe with an RFID tag, it moves between the door and registers the wearer syncing fitness data that is tracked by the shoe, this in turn is returned to a physical output like the same bulletin board but this time through direct physical interaction.

Previously we discussed the many interactions happening in the model, but at the centre much more complex interactions take place. Utilising from the mirror analogy of a utopia this space has been marked $\boldsymbol{U}$ and here is where a virtually private-public yet simultaneously physically private-public interaction takes place. In order to imagine this, levels of permission and trust need to be facilitated and that can only happen if the different interactions allow for major alterations in the nature of information handling. Imagine a scenario where your fitness data is tracked to your FitBit, that in turn sends data to a digital server, which allows access to physical devices to relay that information when and where they wish, now picture going into a gym and seeing a wall light up with your specific information keeping track of your steps and sharing it with you but very openly so others can see and possibly interact with it as well. Such an interaction can only take place when levels of permissions have been allowed over different spaces through policies, regulations, different terms 
and conditions and so on. By making this interaction between user-device-service-institute and so on new heterotopias are dynamically created where the rules differ and thus the device has to operate in that particular way; any change happening in any of those rules reverberates through the entire constellation.

\section{Conclusions}

In this paper we have presented a way of characterising digital and physical interactions by imagining a relationship between spaces and levels of permission explained through a philosophical lens of heterotopias. It can be seen through Figure 3 that the closer one gets to the centre of the Inter-Spatial Interactivity Model the greater the complexity of interactions occur. The increased levels of complexity, which includes increasingly diffused relationships of trust, raise a question into the meaningfulness in how these interactions happen. Interconnectivity between physical and digital interfaces are becoming more and more common with loT surfacing in newer more seemingly efficient forms often as designed artefacts. But the complexity that ensues from these interactions means that a lot of information is either lost, ignored, or deliberately obfuscated. When various previously clear relationships of trust are being altered, is the interaction still worth it to the actor? Are there any measures that can be taken in order to renegotiate this trust or indicated that it has changed?

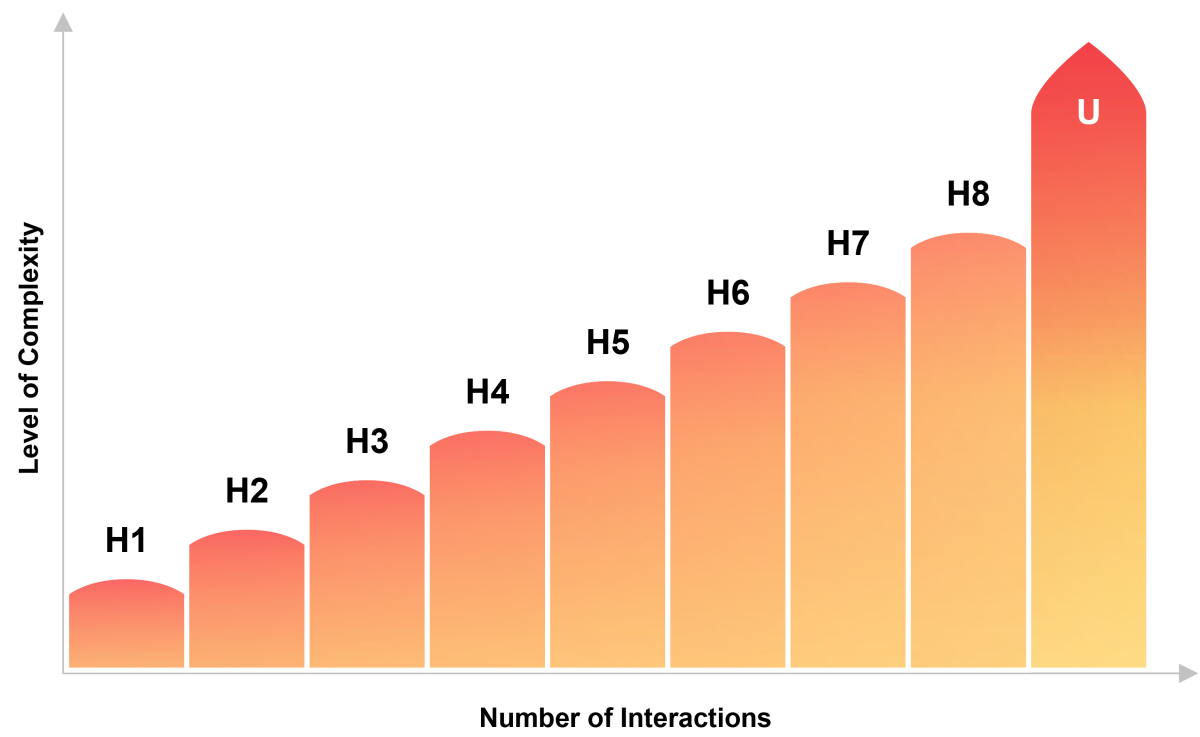

Figure 3. Relationship of number of interaction to level of complexity within Inter-Spatial Interactivity Model

Though Human-Centred Design (HCD) "has become the de facto modus operandi" (Lindley et al., 2017) for designing for loT, concerns have been raised over how this approach "obscure[s] underlying complexities from users". Designers have always affected the "well-being and lives of users and society at large" (Stam \& Eggink, 2014), Stam and Eggink (2014) have argued for the use of philosophy in design saying that "encouraging designers to engage with deeper philosophical issues about their practice and research will contribute to a more profound understanding of design" (p. 5), this approach of using philosophical constructs as a support structure to look at the larger picture of a design problem can allow designers to be "more aware of the power of design and help them to envision how their designs can contribute to a more desirable future" (p. 5). The use of Michel Foucault's philosophies as a basis for studying complex digital/physical interactions is in effect a way to help better understand HCD pitfalls when designing for these kinds of interactions. Using the above model in conjunction with philosophical constructs such as Object-Oriented Ontology (OOO) it is possible to use philosophy further as a tool to help in design research with relation to dissecting the inevitable messiness that is associated with digital and physical interactions seen in loT devices. 
Lindley et al. (2017) have referred to the interaction between people and technologies as a "Pandora's box of possibility" being opened, this model and approach allows to mitigate these possibilities and force us to focus on individual interactions to see them as acting indifferently to their surroundings raising questions for other areas of study that could benefit from the information extracted in this process. One particular direction to move on from here could be looking at where value for stakeholders lies in this model? When seeing physical/digital interactions happen in this fashion, is it possible to further utilise it to see how design could be used to benefit or contest any political or economical interests? Foucault once defined discourse as "going outside of oneself ultimately to find oneself" (Foucault, 1987, p. 16), using philosophy as a discourse building activity to better understand the complexity of design problems - as in this case giving meaning and purpose to objects and spaces in order to understand physical/digital interactions-can prove as a strong tool in a design researchers belt and possibly help in establishing the need for meaningful interactions to be taken into consideration through larger perspectives as well as individual ones.

\section{References}

Brooker, P. (1999). Cultural theory: A glossary. Arnold.

Cole, A. (2013). The Call of Things A Critique of Object-Oriented Ontologies. The Minnesota Review, 2013(80), $106-118$.

Coulton, P. (2015). Playful and gameful design for the Internet of Things. In More Playful User Interfaces (pp. 151-173). Springer.

Coulton, P. (2017). Sensing Atoms and bits. Sensory Arts and Design, 189.

Foucault, M. (1967). Des Espace Autres (Of Other Spaces), Heterotopias. Architecture/Mouvement/Continuité. Foucault, M. (1987). Foucault Blanchot.

Hetherington, K. (2002). The Badlands of Modernity. Routledge.

Leedy, P., \& Ormrod, J. (2010). Practical Research planning and design 9th edition Boston: Pearson Education International.

Lindley, J. G., Coulton, P., \& Cooper, R. (2017). Why the internet of things needs Object Orientated Ontology. Lindley, J., \& Coulton, P. (2017). On the Internet No Everybody Knows You're a Whatchamacallit (or a Thing).

Milgram, P., Takemura, H., Utsumi, A., \& Kishino, F. (1995). Augmented reality: A class of displays on the reality-virtuality continuum, 1994. SPIE Proceedings Tele-Manipulator and Telepresence Technologies, Boston, MA.

Monk, J. (1997). The Digital Unconscious. In J. Wood (Ed.), The Virtual Embodied: Practices, Theories and the New Technologies.

Muratovski, G. (2015). Research for designers: A guide to methods and practice. Sage.

Rymarczuk, R., \& Derksen, M. (2014). Different spaces: Exploring Facebook as heterotopia. First Monday, 19(6). Retrieved from http://firstmonday.org/ojs/index.php/fm/article/view/5006

Slane, A. (2007). Democracy, social space, and the internet.

Smith, D. W. (2016). Phenomenology. In E. N. Zalta (Ed.), The Stanford Encyclopedia of Philosophy (Winter 2016). Metaphysics Research Lab, Stanford University. Retrieved from https://plato.stanford.edu/archives/win2016/entries/phenomenology/

Stam, L., \& Eggink, W. (2014). Why Designers and Philosophers should meet in School. In. The Design Society. Tuan, Y.-F. (1977). Space and Place. University of Minnesota Press.

Varnelis, K., \& Friedberg, A. (2008). Place: The networking of public space. Networked Publics.

About the Authors:

Haider Ali Akmal is a PhD Design student and practicing visual artist at Imagination Lancaster, Lancaster University. His research focuses on the intricacies of human experiences through intimate digital and physical interactions using Speculative Design as an exploratory medium.

Paul Coulton is the Chair of Speculative and Game Design in the open and exploratory design-led research studio Imagination Lancaster. He uses a research through design approach to creative fictional representations of future worlds in which emerging technologies have become mundane. 